\title{
Accurate and effective computation of the multi-phonon nonradiative transition
}

\author{
Su-Huai Wei ${ }^{*}$ \\ Beijing Computational Science Research Center, Beijing 100193, China \\ Received April 7, 2020; accepted April 24, 2020; published online May 19, 2020
}

Citation: $\quad$ S.-H. Wei, Accurate and effective computation of the multi-phonon nonradiative transition, Sci. China-Phys. Mech. Astron. 63, 117331 (2020), https:// doi.org/10.1007/s11433-020-1570-y

Nonradiative carrier recombination at a defect or impurity level can reduce the collection of photo-generated carriers and suppress luminescence. Therefore, understanding the underlying mechanism and providing an accurate theoretical prediction of the carrier capture rate (CCR) of a defect in a semiconductor are important for both semiconductor physics and device engineering [1-5]. The ab initio calculation of the CCR is time-consuming because it requires an accurate calculation of the defect-induced electron-phonon coupling matrix in a large supercell containing a single point defect. It becomes possible only recently after Wang's group [3] developed an effective method to calculate the defect-induced electron-phonon coupling matrix [3-5]. However, almost all current approaches are based on the assumption that the phonon modes before and after the electronic transition are the same; while in reality, for the defects with different charge states before and after the transition, their phonon modes could be quite different, especially for the localized deep-level defects that often involve large local distortions associated with the charge state of the defect [5]. Therefore, it is crucial to investigate how the so-called anharmonic effect affects the calculation of the CCR.

In this paper entitled "Anharmonic multi-phonon nonradiative transition: An ab initio calculation approach" [1], the authors provide an analysis of Huang's original non- radiative multi-phonon (NMP) theory and demonstrate that it provides an analytical expression for the coupling constant in the widely used Marcus theory [3]. Most importantly, the previously ignored anharmonic effects are corrected by taking into account local phonon-mode variations for different charge states of a defect, and a detailed calculation procedure is provided. They show that after the correction, the calculated CCRs for the deep-level defects in prototype $\mathrm{GaN}$ and $\mathrm{SiC}$ are in better agreement with theexperimental results.

Because the nonradiative carrier recombination plays a key role in understanding the semiconductor device physics [35], and the direct measurement of the CCR in experiment is still quite challenging, the accurate calculation of CCR becomes especially critical. This work has made a significant and timely contribution in this field [1]. This will certainly help us improve our understanding of the semiconductor defect physics and optoelectronic device performance.

1 Y. Xiao, Z.-W. Wang, L. Shi, X. Jiang, S.-S. Li, and L.-W. Wang, Sci. China-Phys. Mech. Astron. 63, 277312 (2020).

2 K. Huang, and A. Rhys, Proc. R. Soc. Lond. A 204, 406 (1950).

3 L. Shi, and L. W. Wang, Phys. Rev. Lett. 109, 245501 (2012).

4 A. Alkauskas, Q. Yan, and C. G. Van de Walle, Phys. Rev. B 90, 075202 (2014), arXiv: 1407.4197.

5 J. H. Yang, L. Shi, L. W. Wang, and S. H. Wei, Sci. Rep. 6, 21712 (2016).

*Corresponding author (email: suhuaiwei@csrc.ac.cn) 